\title{
Matriciamento em Saúde Mental: análise do cuidado às pessoas em sofrimento psíquico na Atenção Básica
}

\author{
Matrix Support in Mental Health: analysis of care provided \\ to people in psychic distress in Primary Care
}

Giselle Soares Fagundes (https://orcid.org/0000-0001-6801-2874 ) ${ }^{1}$

Monica Rodrigues Campos (https://orcid.org/0000-0002-7443-5977) ${ }^{1}$

Sandra Lúcia Correia Lima Fortes (https://orcid.org/0000-0002-9918-1555) ${ }^{2}$
${ }^{1}$ Departamento de Ciências Sociais, Escola Nacional de Saúde Pública Sergio Arouca, Fundação Oswaldo Cruz. R. Leopoldo Bulhões 1.480 , Manguinhos.

21041-210 Rio de Janeiro RJ Brasil. gisellesfagundes@ gmail.com

${ }^{2}$ Faculdade de Ciências Médicas, Universidade do Estado do Rio de Janeiro. Rio de Janeiro RJ Brasil.

\begin{abstract}
The prevalence of emotional distress in the population attended by the teams of the Family Health Strategy is high, however the training of the Primary Care professionals in Mental Health care is inadequate. Matrix Support, provided through the Family Health Support Centers, is considered fundamental in the provision of such care. The scope of this study is to assess the training in mental health in primary care through matrix support actions in mental health. A quantitative, cross-sectional study was conducted in the secondary database of the 2012 National Program for the Enhancement of the Access to and the Quality of Primary Health Care. The associations between many matrix support activities in mental health and the development of comprehensive care strategies were analyzed. It revealed that $60 \%$ of the teams carry out Matrix Support actions in Mental Health, and qualified care strategies in mental health are performed at least twice as often when those activities are present. The most significant activities are discussion of cases, joint physician appointments, and the creation of therapeutic projects. The conclusion drawn is that this study provides quantitative evidence that the development of shared actions is decisive for the provision of mental health care in primary care.
\end{abstract}

Key words Matrix support, Mental health, Primary care
Resumo A prevalência de sofrimento emocional na população atendida pelas equipes da Estratégia de Saúde da Família (ESF) é alta, mas a capacitação dos profissionais da Atenção Básica (AB) para o cuidado em Saúde Mental (SM) é inadequada. O Apoio Matricial (AM), através dos Núcleos de Apoio à Saúde da Família (NASF), tem sido considerado como fundamental na qualificação deste cuidado. Tem-se como objetivo analisar a qualificação do cuidado em SM na AB através das ações de Apoio Matricial em Saúde Mental (AMSM). Trata-se de estudo quantitativo (corte transversal) em base de dados secundária do Programa Nacional de Melhoria do Acesso e da Qualidade da Atenção Básica (PMAQ-AB), de 2012. Foram analisadas as associações entre a realização das diversas ações de matriciamento em SM e o desenvolvimento das estratégias de cuidado integral, avaliadas no instrumento. Verificou-se que $60 \%$ das equipes realizam ações de AMSM e que as estratégias de cuidado qualificadas em SM são realizadas pelo menos duas vezes mais quando estas ações estão presentes, destacando-se as ações de discussões de caso, consultas conjuntas e construção de projetos terapêuticos (PTS). Este estudo traz evidências quantitativas de que o desenvolvimento de ações compartilhadas é determinante para qualificação do cuidado em SM na AB.

Palavras-chave Apoio matricial, Saúde mental, Atenção básica 


\section{Introdução}

O Brasil passa por um processo de transição epidemiológica, desde a década de 1960, que se caracteriza pela predominância de doenças não-transmissíveis $(66,3 \%)$ em substituição aos adoecimentos e mortes relacionadas às doenças transmissíveis $(23,5 \%)$. Os transtornos mentais, inseridos no primeiro contexto, representam grande proporção das causas de adoecimento e incapacidade na população brasileira, superando até mesmo as doenças cardiovasculares e o câncer ${ }^{1}$.

Em uma perspectiva global, a Organização Mundial de Saúde (OMS) ${ }^{2}$ sinaliza que mais de 450 milhões de pessoas sofrem em decorrência de transtornos mentais, que estes representam quatro entre as dez principais causas de incapacidade no mundo todo e que uma a cada quatro pessoas no mundo desenvolverão ao longo de suas vidas alguma forma de adoecimento psíquico. Apesar de estimativas demonstrarem que aos transtornos mentais pesam cerca de $12 \%$ das doenças mundiais ${ }^{2}$, os recursos financeiros direcionados ao tratamento de tais morbidades não ultrapassam a marca de $1 \%$ na maioria dos países e há grande ausência de políticas públicas específicas de saúde mental em $40 \%$ dos países.

A lacuna de tratamento verificada pode superar a marca dos 75\% nos países mais pobres ${ }^{3}$ e a integração de cuidados em saúde mental na $\mathrm{AB}$ colaboram significativamente para a amenizar esta realidade ${ }^{4}$. A OMS apontou, em relatório sobre a saúde no mundo, como primeira recomendação que se desenvolvam qualitativamente as políticas de saúde mental para a população proporcionar cuidado em saúde mental nos dispositivos da atenção primária ${ }^{2}$. Aproximadamente $80 \%$ da população terá, pelo menos, um atendimento com seu médico de família durante um ano, o que torna a $\mathrm{AB}$ um nível privilegiado para identificação de necessidades de cuidado e tratamento.

No Brasil, a AB ganhou destaque no sistema de saúde a partir da década de 1990, quando passou a redirecionar o modelo de assistência no Brasil $^{5}$. Com a prerrogativa de ser a coordenadora da rede de saúde e promover cuidado integral, longitudinal e resolver $80 \%$ dos problemas de saúde da população do território de sua abrangência $^{6}$, a AB já se depara com esta questão. Estudos mostram que mais de $50 \%$ dos pacientes em atendimento em unidades da ESF apresentam sofrimento emocional importante e $40 \%$ também preenchem critério para serem considerados casos suspeitos de transtornos depressivo-ansio$\operatorname{sos}^{7}$.

Contudo, observam-se algumas dificuldades no cuidado em SM na AB como a falta de capacitação dos profissionais da $\mathrm{AB}$ na intervenção aos sujeitos em sofrimento psíquico. Apesar de se sentirem preparados para o acolhimento do sofrimento apresentado, não possuem ferramentas teóricas e práticas para uma intervenção que considerem ser de qualidade e que promova cuidado para além do emergencial, prevalecendo sentimentos de medo e incapacidade em relação ao sofrimento psíquico ${ }^{8,9}$. Há também a baixa detecção de transtornos mentais, apresentando uma lacuna que pode alcançar 50\% dos casos, além dos frequentes erros diagnósticos ${ }^{10}$ e o uso inadequado de psicotrópicos, como por exemplo, a prescrição inapropriada de benzodiazepínicos.

O cuidado colaborativo (collaborative care) emerge como proposta de intervenção no desenvolvimento de práticas que visam proporcionar relações de trabalho mais estreitas entre profissionais especializados e da atenção primária, para integrar os cuidados em saúde mental na $\mathrm{AB}^{11}$. Os cuidados colaborativos, além de se mostrarem muito eficazes no tratamento dos transtornos mentais comuns, também são menos onerosos aos sistemas de saúde. Eles dizem respeito ao compartilhamento de conhecimento, competências, recursos e tomadas de decisão, vêm sendo realizados ao redor do mundo e tem na experiência desenvolvida no Canadá ${ }^{12}$ grande inspiração.

O modelo de cuidados colaborativos implementado no Brasil é o Apoio Matricial (AM), termo utilizado inicialmente no final do século $\mathrm{XX}^{13}$. Propõe um novo modo de organização da saúde, com a estruturação de novos processos de trabalho que envolvam diferentes equipes no cuidado aos sujeitos, numa perspectiva de corresponsabilização dos casos, integrando diferentes especialidades e níveis de cuidado. Como uma concepção inovadora em saúde, o matriciamento é o processo de trabalho em que especialistas prestam suporte para as equipes da $\mathrm{AB}$ de modo a produzir melhor assistência aos usuários atendidos neste nível de atenção, por intermédio de ações terapêuticas conjuntas e atividades pedagógicas, assumindo assim um caráter técnico-pedagógico $^{14}$.

O matriciamento é uma prática interdisciplinar e a proposta é que profissionais da $\mathrm{AB}$ e especialistas de diferentes áreas atuem juntos, com vistas a qualificação do cuidado fornecido na $A B$ e a diminuição de encaminhamentos desnecessários $^{15}$, sem de fato haver uma demanda clara- 
mente estabelecida que justifique a atenção especializada. A organização do trabalho deve ser dar de modo a fomentar parcerias, onde o ambiente favoreça que os profissionais possam trocar seus saberes, ampliando assim suas concepções sobre adoecimento e formas de tratamento aos sujeitos.

Há, desta maneira, um novo modo de produzir saúde com o compartilhamento do cuidado entre duas ou mais equipes que tem o potencial de modificar não só o processo saúde-doença, mas incide também sobre a realidade das equipes envolvidas e nas comunidades ${ }^{14}$. Sua efetivação exige a criação de novas tecnologias de trabalho que se pautem na construção do vínculo, permitam a apreensão das singularidades de cada indivíduo e também os contextos em que elas estão inseridas.

Embora qualquer unidade de saúde possa realizar o matriciamento, os Núcleos de Apoio à Saúde da Família (NASF) são equipes em que realizar o apoio matricial é parte intrínseca de seu trabalho. Eles foram implementados em $2008^{16}$ integrando e dando mais robustez à $\mathrm{AB}$ prestada no Brasil, seguindo suas mesmas diretrizes ${ }^{17}$. O trabalho realizado pelos NASF se traduz em ações clínicas conjuntas com os usuários e atividades de educação permanente com os profissionais da ESF, que de maneira frequente ocorrem simultaneamente. Ressalta-se que o AM se implementa a partir de uma pluralidade de novas tecnologias em saúde tais como: consultas conjuntas, discussão de casos, construção de projeto terapêutico; realização de intervenções específicas de SM na $\mathrm{AB}$ (tais como grupos terapêuticos); ou ainda, para fortalecimento da integração entre o nível especializado e a $\mathrm{AB}$.

Contudo, a realização do presente estudo ancora-se no fato de que se verificam situações onde ações são realizadas e nomeadas como apoio matricial de maneira equivocada ${ }^{15}$. Ainda persiste a ideia de que o suporte especializado é mais uma oferta na cesta de serviços das unidades básicas, funcionando sob a lógica de encaminhamentos, com agenda para marcação de atendimentos com especialistas, em que predominam relações verticais entre os profissionais ${ }^{18}$.

Ademais, diante da relevância do tema para a saúde pública, apesar da existência de uma vasta quantidade de produções científicas que se debruçam a estudar a integração dos cuidados em SM na $A B$, por meio do apoio matricial, não foram encontrados artigos científicos publicados com metodologia quantitativa acerca do impacto do apoio matricial no cuidado em SM na $\mathrm{AB}$, no Brasil.
Deste modo, o presente artigo propõe a realização de uma pesquisa quantitativa que analise os efeitos das ações de matriciamento em saúde mental no cuidado aos sujeitos em sofrimento psíquico na $\mathrm{AB}$.

\section{Metodologia}

\section{Fonte de Informação}

Este estudo usa como banco de dados uma fonte de informação secundária que é o Programa Nacional de Melhoria do Acesso e da Qualidade da Atenção Básica (PMAQ-AB), em seu primeiro ciclo de aplicação (2012). O PMAQ-AB é, atualmente, a principal ferramenta de avaliação e monitoramento das ações da atenção básica que abrange todo país. É instrumento de consolidação da Atenção Básica ao inaugurar um elemento qualitativo ao financiamento, pois certifica as equipes da $\mathrm{AB}$ de acordo não só com as características burocráticas do funcionamento da unidade de saúde, mas também levando-se em conta a qualidade dos serviços e ações prestadas, ouvindo gestores, trabalhadores e os usuários ${ }^{19}$.

O PMAQ-AB, em 2012, era divido em três módulos: o primeiro, direcionado aos gestores (Observação da Unidade de Saúde); o segundo, aos profissionais (Entrevista com Profissional da Equipe de Atenção Básica e Verificação de Documentos na Unidade de Saúde); e o terceiro, aos usuários (Entrevista na Unidade de Saúde com Usuário). Este estudo terá como base de observação os dados obtidos por questões trazidas no módulo II, uma vez que é somente neste módulo que podem ser analisados aspectos referentes ao desenvolvimento do apoio matricial. O módulo I aborda questões sobre a infraestrutura e insumos das unidades de saúde e o módulo III sobre o acesso aos usuários às unidades, profissionais e medicamentos. Nenhum destes analisa aspectos de processo de trabalho, onde está incluído o apoio matricial.

\section{Variáveis e Indicadores}

Para iniciar a pesquisa foi criada a variável "Apoio Matricial em Saúde Mental" para caracterizar as equipes de NASF, pois esta definição não constava no banco de dados original do PMAQ-AB. Para defini-la utilizou-se como critério a presença no NASF de pelo menos um profissional das seguintes categorias: psicólogo, assistente social, psiquiatra e terapeuta ocupacional. Tais 
categorias são elencadas no Guia Prático de Matriciamento em Saúde Mental como matriciadores em saúde mental na atenção básica ${ }^{15}$.

No PMAQ-AB são definidas oito ações de apoio matricial: consultas clínicas (ação 1); discussão dos casos (ação 2); ações clínicas compartilhadas (ação 3); construção de PTS (ação 4); educação permanente (ação 5); discussão sobre o processo de trabalho (ação 6); ações territoriais (ação 7) e visitas domiciliares (ação 8). As estratégias de cuidado ao usuário com transtorno mental são definidas por quatro variáveis, quais sejam: consulta específica com tempo maior; registro da história de vida; oferta de algum tipo de atendimento em grupo e atendimento com profissionais de saúde mental - que se referem à qualificação do cuidado ofertado pela equipe da ESF. Consideramos então que estudar as associações entre as ações de matriciamento e as estratégias de cuidado permitiria o entendimento da influência daquelas na qualificação do cuidado em SM prestado na $\mathrm{AB}$.

Variáveis pertencentes a outras categorias foram também utilizadas para análise por se referirem diretamente aos cuidados da área de saúde mental. São elas: a existência de protocolos que definam diretrizes terapêuticas para o acolhimento das demandas espontâneas e urgências identificadas como sendo relacionadas a problemas de saúde mental; a preparação das equipes no trato com os usuários com transtorno mental no cotidiano; a existência de registro dos casos mais graves de usuários com transtorno mental; o registro de casos de usuários com necessidades decorrentes do uso de crack, álcool e outras drogas; registro dos usuários que fazem uso crônico de benzodiazepínicos; bem como, a identificação das equipes que realizam ações direcionadas à diminuição da dose de benzodiazepínicos.

Para efeito de análise e discussão da temática, a partir dos dados optou-se por agregar as dez variáveis referentes às estratégias de cuidado, descritas anteriormente, formando quatro novas categorias, elaboradas conforme a seguinte agregação:

Qualificação da Clínica (QC):

- consulta específica com tempo maior;

- registro da história de vida.

Desenvolvimento de Novas Tecnologias (DNT):

- oferta de algum tipo de atendimento em grupo;

- atendimento com profissionais de saúde mental;

- a equipe realiza ações para pessoas que fazem uso crônico de benzodiazepínicos e necessitam desmame (diminuição da dose)?
Gestão do Caso (GC):

- a equipe possui protocolos com definição de diretrizes terapêuticas para acolhimento à demanda espontânea/urgência para problemas relacionados à saúde mental?;

- a eSF teve preparação para o atendimento dos usuários com transtorno mental?

Qualificação do Registro (QR):

- a eSF possui registro dos casos mais graves de usuários com transtorno mental?;

- há registro de usuários com necessidades por uso de crack/álcool/outras drogas?;

. a equipe possui registro dos usuários em uso crônico de benzodiazepínicos?

De modo a identificar a existência ou não de associação (influência) entre tais ações de matriciamento e as estratégias de cuidado será aplicado o teste qui-quadrado, ao nível de significância de 5\%, utilizando o programa SPSS, considerando-se a presença ou não de apoio matricial em saúde mental (AMSM). Para mensurar e analisar a magnitude e direção do efeito de cada uma das ações de matriciamento separadamente em relação às estratégias de cuidado serão calculados seus Odss Ratio (OR), e respectivos intervalos de confiança, em nível "agregado" e "desagregado".

Cabe ressaltar que todas as equipes voluntárias ao $\mathrm{PMAQ}-\mathrm{AB}$ declararam receber apoio matricial em alguma especialidade, contudo, neste estudo, foi investigado especificamente apenas o AMSM.

\section{Resultados}

Em 2012 participaram do PMAQ-AB 15.120 eSf e destas 9.057 informaram realizar ações de AMSM, ou seja, 60\% do total das equipes investigadas formam o universo de análise deste estudo. Dentre estas equipes que realizam apoio matricial em saúde mental, a "ação 1" foi a mais frequentemente realizada pelas eSF (consultas clínicas), realizada por $85 \%$ das equipes, enquanto que a menos executada foi a "ação 6" (discussão sobre o processo de trabalho), apenas em $24,5 \%$ (percentual coluna na Tabela 1). Em relação ao efeito quando há ou não AMSM, observa-se que todas as oito ações têm maior chance de serem realizadas quando têm-se também a presença do AMSM. Por exemplo, para a "ação 8" (visitas domiciliares) é possível notar que ao se desconsiderar o AMSM tem-se cerca de $60 \%$ de realização desta, porém quando há AMSM esta proporção atinge aproximadamente $80 \%(\mathrm{OR}=2,72$ - Tabela 1$)$.

No tocante às estratégias de cuidado, observa-se que o "Desenvolvimento de Novas Tecno- 
Tabela 1. Distribuição proporcional das ações de matriciamento e estratégias de cuidados segundo a eSF ter ou não apoio matricial em saúde mental (profissionais do NASF). Brasil, 2012.

\begin{tabular}{|c|c|c|c|c|c|c|}
\hline \multirow{3}{*}{ Variáveis de investigação } & \multicolumn{6}{|c|}{ Apoio Matricial em Saúde Mental - NASF $\left(^{*}\right)$} \\
\hline & \multirow{2}{*}{$\begin{array}{c}\text { Sim } \\
\% \text { Col }\end{array}$} & \multirow{2}{*}{$\begin{array}{c}\text { Não } \\
\text { \%Col }\end{array}$} & \multirow{2}{*}{$\begin{array}{c}\text { Total } \\
\% \text { Col }\end{array}$} & \multirow{3}{*}{ OR } & \multicolumn{2}{|c|}{ IC 95\% } \\
\hline & & & & & \multirow{2}{*}{ Inf } & \multirow{2}{*}{ Sup } \\
\hline Ações de matriciamento & $\mathrm{N} 1=9.057$ & $\mathrm{~N} 2=6.063$ & $\mathrm{~N} 1+\mathrm{N} 2=15.120$ & & & \\
\hline $\begin{array}{l}\text { Ação } 1 \text { - Consultas clínicas de pacientes da } \\
\text { Atenção Básica }(\mathrm{AB})\end{array}$ & 87,6 & 81,0 & 85,0 & 1,65 & 1,51 & 1,80 \\
\hline $\begin{array}{l}\text { Ação } 2 \text { - Discussão de casos clínicos, eventos } \\
\text { sentinelas, casos difíceis e desafiadores }\end{array}$ & 77,0 & 61,1 & 70,6 & 2,13 & 1,98 & 2,29 \\
\hline $\begin{array}{l}\text { Ação } 3 \text { - Ações clínicas compartilhadas com } \\
\text { profissionais da equipe de } A B\end{array}$ & 76,8 & 59,5 & 69,8 & 2,25 & 2,10 & 2,42 \\
\hline $\begin{array}{l}\text { Ação } 4 \text { - Construção conjunta de projetos } \\
\text { terapêuticos com profissionais da } \mathrm{AB}\end{array}$ & 61,1 & 39,5 & 52,5 & 2,40 & 2,25 & 2,57 \\
\hline $\begin{array}{l}\text { Ação } 5 \text { - Atividades de educação permanente } \\
\text { conjuntamente com profissionais da } \mathrm{AB}\end{array}$ & 67,8 & 46,3 & 59,2 & 2,43 & 2,28 & 2,60 \\
\hline $\begin{array}{l}\text { Ação } 6 \text { - Discussões sobre o processo de trabalho } \\
\text { da Equipe de Atenção Básica e também do } \\
\text { próprio apoio matricial }\end{array}$ & 24,5 & 18,1 & 21,9 & 1,46 & 1,35 & 1,59 \\
\hline $\begin{array}{l}\text { Ação } 7 \text { - Organização de intervenções no } \\
\text { território em conjunto com sua equipe }\end{array}$ & 70,0 & 50,7 & 62,3 & 2,26 & 2,12 & 2,42 \\
\hline $\begin{array}{l}\text { Ação } 8 \text { - Visitas domiciliares com os profissionais } \\
\text { da sua equipe }\end{array}$ & 78,2 & 56,8 & 69,6 & 2,72 & 2,53 & 2,92 \\
\hline \multicolumn{7}{|l|}{ Estratégias de cuidado } \\
\hline Qualificação da clínica (QC) & 57,4 & 46,8 & 52,4 & 1,52 & 1,43 & 1,62 \\
\hline Desenvolvimento de novas tecnologias (DNT) & 73,0 & 56,3 & 65,1 & 2,09 & 1,96 & 2,23 \\
\hline Gestão do caso (GC) & 55,5 & 38,8 & 47,7 & 1,96 & 1,85 & 2,09 \\
\hline Qualificação do registro (QR) & 64,2 & 53,1 & 59,0 & 1,57 & 1,48 & 1,67 \\
\hline
\end{tabular}

${ }^{\star}$ Todas as associações (OR's) são estatisticamente significantes com p_valor $<5 \%$.

Fonte: Elaboração própria com base no Programa Nacional de Melhoria do Acesso e da Qualidade da Atenção Básica (PMAQ-AB), em seu primeiro ciclo de aplicação (2012).

logias" é a mais realizada, em $65,1 \%$ das equipes, enquanto que a "Gestão do Caso" é realizada em apenas $47,7 \%$ das equipes, sendo a menos realizada. Estas duas categorias também são as que sofrem maior influência da realização ou não do AMSM. O DNT é realizado por $56,3 \%$ das eSF quando não há AMSM e esta percentagem salta para $73 \%$ na presença do $\mathrm{AM}(\mathrm{OR}=2,09)$, como é demonstrado na Tabela 1.

O principal destaque da Tabela 2, que traz a distribuição das razões de chance (OR) entre as estratégias de cuidados e as ações de matriciamento com e sem AMSM, diz respeito ao fato de que todas as associações entre as oito ações de apoio matricial com as quatro categorias de estratégias de cuidado mostraram-se positivas e estatisticamente significantes (p_valor $<5 \%$ ), apontando para ocorrência de uma melhor atenção ao cuidado em saúde mental na ESF quando estas ações são realizadas. Além disso, é importante ressaltar que tais associações se expressaram de forma mais acentuada, ou seja, mais fortes (com maiores valores) na situação na qual contou-se com o AMSM, à exceção da "ação 1" (consultas clínicas), frente à estratégia de cuidado DNT $\mathrm{OR}=1,54 \times \mathrm{OR}=1,71$ - em situação com apoio matricial $\mathrm{x}$ sem apoio matricial, respectivamente.

Quanto à "Qualificação da Clínica", sem apoio matricial, ao realizar-se a "ação 1" (consultas clínicas), observa-se uma associação invertida e tênue (bem próximo de 1 com $\mathrm{OR}=0.96$ ), mais uma vez apontando para a força do AMSM $(\mathrm{OR}=1,28)$. Considerando-se apenas as eSF respondentes que declararam ter AMSM, ao comparar-se o efeito das oito ações conjuntamente sobre as quatro categorias de estratégia de cuidado, observa-se que a "ação 2" (discussão de casos clínicos) foi a que se mostrou como a mais eficiente (associada com maior magnitude) de todas as estratégias de cuidado. Esta foi seguida de perto, quanto à eficiência pela "ação 4" (construção de PTS) e a "ação 3" (ações clínicas compartilhadas). 
Por outro lado, a "ação 1" (consultas clínicas), foi a que teve menor efeito, embora positivo, conforme apresentado na Tabela 2.

Devido ao fato de que as ações 2 (discussão de casos), 3 (ações clínicas compartilhadas) e 4 (construção de PTS) terem se mostrado as mais eficientes na obtenção de melhores resultados nas estratégias de cuidado, essas ações foram analisadas de forma mais detalhada nas Tabelas 3, 4 e 5). Os resultados apresentam os efeitos de cada uma das referidas ações em particular sobre as estratégias de cuidado, considerando as categorias em dois níveis de análise: agregadas e desagregadas.

De modo geral, nas três tabelas apresentadas a seguir temos que as ações nelas destacadas têm maiores efeitos nas seguintes estratégias de cuidado: Desenvolvimento de Novas Tecnologias e Gestão do Caso, com indicadores de associação de forte magnitude. Observa-se três vezes mais chance de ocorrência dessas estratégias ao realizar-se discussão de casos graves, ações clínicas compartilhadas, bem como ao fazer-se a construção conjunta do PTS. Além disso, descendose no nível de análise mais desagregado dessas estratégias, verifica-se que a maior influência de todas as ações é sobre a "oferta de algum tipo de atendimento em grupo" ( $\mathrm{OR}=4,15$ para a ação 2 ; $\mathrm{OR}=3,11$ para ação 4 e $\mathrm{OR}=3,64$ para a ação 3).

\section{Discussão}

O panorama geral deste estudo demonstra que pelo menos $60 \%$ das equipes declararam realizar as ações de matriciamento na presença de AMSM, com exceção da "ação 6" (discussões sobre o processo de trabalho e do próprio apoio matricial) realizada por apenas $24 \%$ destas equipes. Já as estratégias de cuidado, são realizadas por pelo menos $55 \%$ das equipes, mas atinge no máximo $73 \%$ das equipes. Todas as ações de matriciamento e estratégias de cuidado são potencializadas quando há a realização do AMSM, apresentando um aumento de duas vezes mais na sua frequência.

Das oito ações de matriciamento, três destacam-se mais quanto a sua influência positiva na qualificação do cuidado: discussão de casos (ação 2), ações clínicas compartilhadas (ação 3) e construção conjunta de PTS (ação 4). Elas são realizadas por $77 \%, 76,8 \%$ e $61,1 \%$ das equipes, respectivamente. Quando tais ações são implementadas, todas as estratégias de cuidado são realizadas pelo menos duas vezes mais - as demais ações de matriciamento também apresentam associações positivas, porém mais fracas.

A discussão de casos (ação 2) é um procedimento altamente rotineiro nas unidades de saúde. Contudo, a riqueza da utilização desta ferramenta, com a presença de profissionais do apoio matricial, encontra-se no fato de uma equipe

Tabela 2. Distribuição das razões de chance (OR) entre as estratégias de cuidados e as ações de matriciamento na atenção básica (ação 1 a 8), em eSF COM e SEM apoio matricial em saúde mental. Brasil, 2012.

\begin{tabular}{|c|c|c|c|c|c|c|c|c|c|}
\hline & Estratégias de cuidado $^{\star}$ & $\begin{array}{l}\text { Ação } \\
1 \text { OR }\end{array}$ & $\begin{array}{l}\text { Ação } \\
2 \text { OR }\end{array}$ & $\begin{array}{l}\text { Ação } \\
3 \text { OR }\end{array}$ & $\begin{array}{l}\text { Ação } \\
4 \text { OR }\end{array}$ & $\begin{array}{l}\text { Ação } \\
5 \text { OR }\end{array}$ & $\begin{array}{l}\text { Ação } \\
6 \text { OR }\end{array}$ & $\begin{array}{l}\text { Ação } \\
7 \text { OR }\end{array}$ & $\begin{array}{l}\text { Ação } \\
8 \text { OR }\end{array}$ \\
\hline \multirow{4}{*}{ 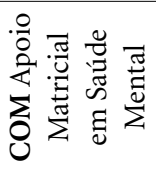 } & Qualificação da clínica & 1,28 & 2,45 & 2,42 & 2,30 & 1,98 & 1,71 & 2,10 & 1,88 \\
\hline & Desenvolvimento de novas tecnologias & 1,54 & 3,27 & 2,82 & 3,10 & 2,24 & 2,10 & 2,29 & 2,04 \\
\hline & Gestão do caso & 1,34 & 3,27 & 2,80 & 3,15 & 2,66 & 2,20 & 2,45 & 1,97 \\
\hline & Qualificação do registro & 1,36 & 2,20 & 2,11 & 2,14 & 1,97 & 1,72 & 2,02 & 1,95 \\
\hline \multicolumn{10}{|c|}{ Estratégias de cuidado* } \\
\hline \multirow{4}{*}{ 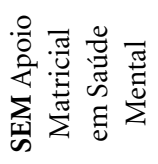 } & Qualificação da clínica & 0,96 & 1,95 & 1,83 & 2,20 & 1,85 & 1,17 & 1,71 & 1,41 \\
\hline & Desenvolvimento de novas tecnologias & 1,71 & 2,38 & 2,21 & 2,47 & 2,02 & 1,60 & 1,86 & 1,67 \\
\hline & Gestão do caso & 1,26 & 2,47 & 2,17 & 2,80 & 2,43 & 1,70 & 2,22 & 1,97 \\
\hline & Qualificação do registro & 1,30 & 1,85 & 1,75 & 1,90 & 1,77 & 1,26 & 1,70 & 1,69 \\
\hline
\end{tabular}

* Todas as associações (OR's) são estatisticamente significantes com p_valor $<5 \%$.

Nota: ação 1- Consultas clínicas de pacientes da Atenção Básica (AB); ação 2 - Discussão de casos clínicos, eventos sentinelas, casos difíceis e desafiadores; ação 3 - Ações clínicas compartilhadas com profissionais da equipe de AB; ação 4 - Construção conjunta de projetos terapêuticos com profissionais da $\mathrm{AB}$; ação 5 - Atividades de educação permanente conjuntamente com profissionais da AB; ação 6 - Discussões sobre o processo de trabalho da equipe de atenção básica e também do próprio apoio matricial; ação 7 Organizam intervenções no território em conjunto com sua equipe; ação 8 - Realizam visitas com os profissionais da sua equipe. 
interdisciplinar se debruçar sobre os casos e com isso fazer emergir diferentes visões sobre o processo de adoecimento dos sujeitos, bem como múltiplas propostas de cuidado. Há, desta forma, uma ampliação do escopo da compreensão sobre os transtornos mentais. É indicado pelo Guia de Matriciamento ${ }^{15}$ um roteiro para discussão de casos em AMSM, que contempla uma vasta gama de elementos, como o problema apresentado, a configuração familiar e a rede social de cada indivíduo. As ações clínicas compartilhadas (ação 3) se configuram como a principal ferramenta do AM e objetivam o desenvolvimento de práticas terapêuticas integrais. Elas integram os caráteres assistenciais e pedagógicos das ações de saúde, ao reunir diferentes atores e saberes em um processo de aprimoramento conjunto ${ }^{20}$. Seu grande potencial está ancorado no fornecimento de bases de atuação para que os profissionais da $\mathrm{AB}$ desenvolvam competências para o cuidado das questões psíquicas apresentados por seus usuários $^{21}$, auxiliando na melhora da qualidade do atendimento prestado e na resolutividade dos casos sem a necessidade de encaminhamentos constantes.

Já o Projeto Terapêutico Singular (PTS) é um instrumento da Clínica Ampliada e resulta de trocas entre a equipe multidisciplinar em que são definidas diretrizes terapêuticas que incluam os recursos das unidades de saúde e intersetoriais, além das ferramentas existentes no território, na família e nos próprios usuários ${ }^{22}$. Nas unidades

Tabela 3. Distribuição proporcional das estratégias de cuidados segundo a realização da ação 2 de matriciamento (discussão de casos clínicos/eventos sentinelas/casos difíceis) em eSF COM apoio matricial em saúde mental. Brasil, 2012.

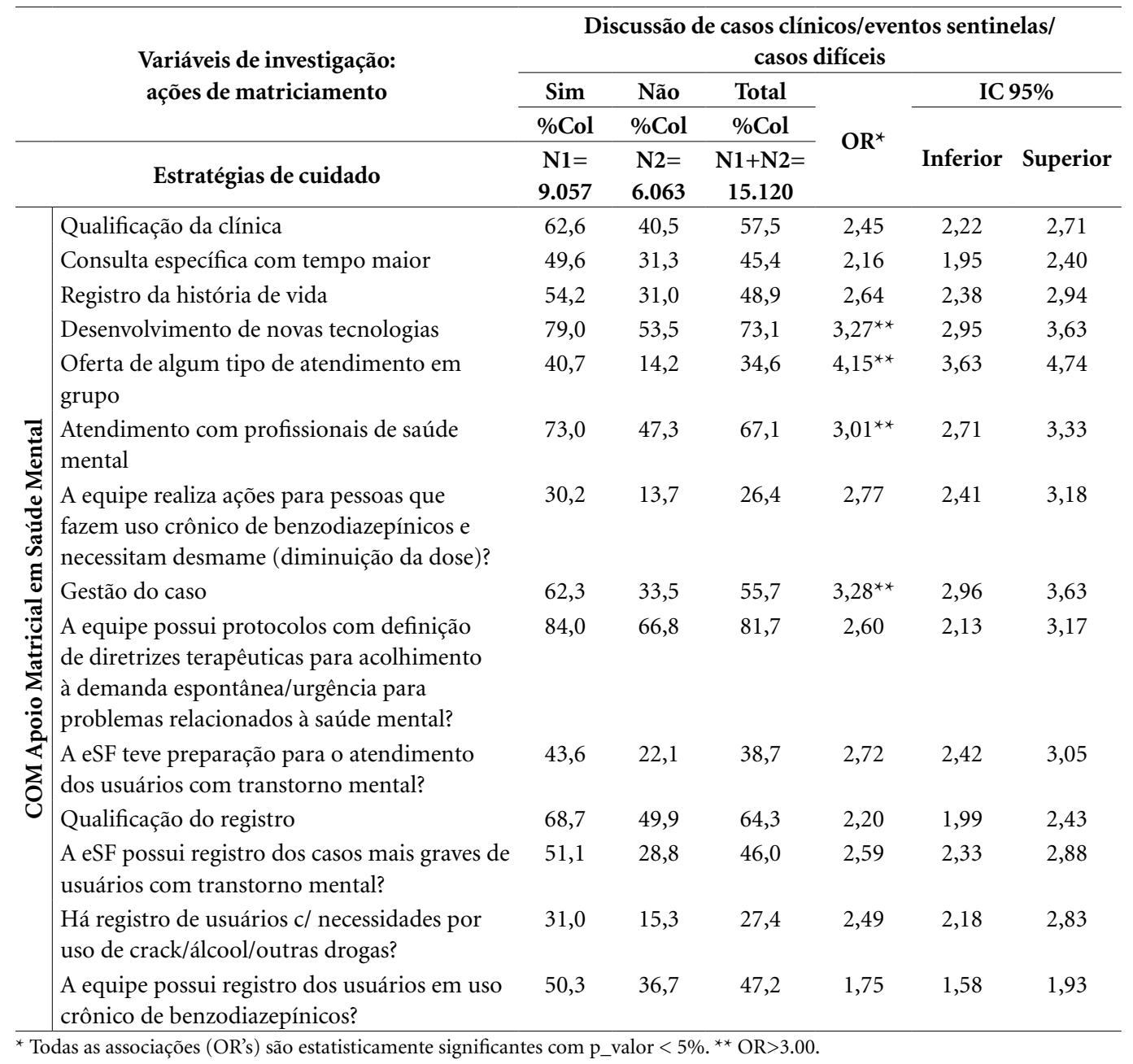


Tabela 4 Distribuição proporcional das estratégias de cuidados segundo a realização da ação 3 de matriciamento (ações clínicas compartilhadas com profissionais da atenção básica) em eSF com apoio matricial em saúde mental. Brasil, 2012.

\begin{tabular}{|c|c|c|c|c|c|c|c|}
\hline \multirow{2}{*}{\multicolumn{2}{|c|}{ Variáveis de investigação: ações de matriciamento }} & \multicolumn{6}{|c|}{ Ações clínicas compartilhadas com profissionais da $\mathrm{AB}$} \\
\hline & & \multirow{3}{*}{$\begin{array}{c}\text { Sim } \\
\% \text { Col } \\
\text { N1= } \\
9.057\end{array}$} & \multirow{3}{*}{$\begin{array}{c}\text { Não } \\
\% \text { Col } \\
\text { N2= } \\
6.063\end{array}$} & \multirow{3}{*}{$\begin{array}{c}\text { Total } \\
\% \text { Col } \\
\text { N1+N2= } \\
15.120\end{array}$} & \multirow{3}{*}{$\mathrm{OR}^{*}$} & \multicolumn{2}{|c|}{ IC $95 \%$} \\
\hline & & & & & & & \\
\hline \multicolumn{2}{|r|}{ Estratégias de cuidado } & & & & & Inferior & Superior \\
\hline \multirow{14}{*}{ 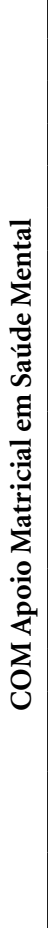 } & Qualificação da Clínica & 62,5 & 40,8 & 57,5 & 2,43 & 2,20 & 2,68 \\
\hline & Consulta específica com tempo maior. & 49,8 & 30,8 & 45,4 & 2,22 & 2,00 & 2,47 \\
\hline & Registro da história de vida. & 54,0 & 32,1 & 48,9 & 2,48 & 2,24 & 2,75 \\
\hline & Desenvolvimento de novas tecnologias & 78,3 & 56,1 & 73,1 & $2,82^{\star *}$ & 2,55 & 3,13 \\
\hline & Oferta de algum tipo de atendimento em grupo. & 39,8 & 17,5 & 34,6 & $3,11^{\star *}$ & 2,75 & 3,52 \\
\hline & Atendimento com profissionais de saúde mental. & 72,2 & 50,0 & 67,1 & 2,60 & 2,35 & 2,88 \\
\hline & $\begin{array}{l}\text { A equipe realiza ações para pessoas que fazem } \\
\text { uso crônico de benzodiazepínicos e necessitam } \\
\text { desmame (diminuição da dose)? }\end{array}$ & 29,8 & 15,3 & 26,4 & 2,39 & 2,09 & 2,73 \\
\hline & Gestão do Caso & 61,6 & 36,3 & 55,7 & $2,80^{\star *}$ & 2,53 & 3,10 \\
\hline & $\begin{array}{l}\text { A equipe possui protocolos com definição } \\
\text { de diretrizes terapêuticas para acolhimento à } \\
\text { demanda espontânea/urgência para problemas } \\
\text { relacionados à saúde mental? }\end{array}$ & 83,9 & 68,9 & 81,7 & 2,35 & 1,93 & 2,85 \\
\hline & $\begin{array}{l}\text { A eSF teve preparação para o atendimento dos } \\
\text { usuários com transtorno mental? }\end{array}$ & 43,0 & 24,1 & 38,7 & 2,38 & 2,12 & 2,66 \\
\hline & Qualificação do registro & 68,5 & 50,7 & 64,3 & 2,17 & 1,92 & 2,34 \\
\hline & $\begin{array}{l}\text { A eSF possui registro dos casos mais graves de } \\
\text { usuários com transtorno mental? }\end{array}$ & 50,3 & 31,7 & 46,0 & 2,18 & 1,69 & 2,41 \\
\hline & $\begin{array}{l}\text { Há registro de usuários c/ necessidades por uso } \\
\text { de crack/álcool/outras drogas? }\end{array}$ & 30,5 & 17,3 & 27,4 & 2,09 & 1,85 & 2,37 \\
\hline & $\begin{array}{l}\text { A equipe possui registro dos usuários em uso } \\
\text { crônico de benzodiazepínicos? }\end{array}$ & 50.5 & 36.1 & 47.2 & 1.81 & 1.63 & 2.00 \\
\hline
\end{tabular}

Fonte: Elaboração própria com base no Programa Nacional de Melhoria do Acesso e da Qualidade da Atenção Básica (PMAQ-AB), em seu primeiro ciclo de aplicação (2012).

de $\mathrm{AB}$, a construção conjunta do PTS envolvendo as equipes da ESF e NASF revela-se uma tecnologia inovadora e particularmente indicada nos casos mais graves e complexos ${ }^{23}$.

Importante destacar que o Desenvolvimento de Novas Tecnologias e a Gestão do Caso são as estratégias de cuidado em que se verifica uma maior influência do matriciamento na sua realização. Ou seja, tais estratégias são implementadas mais vezes na ocorrência de todas as ações de AMSM, sendo realizadas três vezes mais associadas às ações 2 (discussão dos casos) e 4 (construção de PTS), e pouco mais de duas vezes e meia associadas à ação 3 (ações clínicas compartilhadas).

Em análise num nível "desagregado" da categoria Desenvolvimento de Novas Tecnologias, observa-se que as três ações destacadas afetam, de maneira regular, cada estratégia de cuidado, mas têm influência mais efetiva sobre a oferta de atendimento em grupo. Desta forma, pode-se afirmar que o AMSM proporciona uma maior oferta de atividades em grupo aos usuários em sofrimento psíquico atendidos na $\mathrm{AB}$, chegando a ser oferecido quatro vezes mais na presença de AMSM. Esta constatação é de suma relevância um vez que a participação em grupos tem alto potencial transformador e pode auxiliar as pessoas com transtorno mental na criação de novos vínculos sociais, em sua melhor circulação pelo território e no desenvolvimento de maior autonomia em seu próprio tratamento ${ }^{24}$.

$\mathrm{O}$ atendimento com profissionais de saúde mental aumenta em pelo menos duas vezes e meia, o que demostra que o AMSM proporciona mais acesso da população à profissionais 
Tabela 5. Distribuição proporcional das estratégias de cuidados segundo a realização da ação 4 de matriciamento (construção conjunta dos Projetos Terapêuticos Singulares) em eSF com apoio matricial em saúde mental. Brasil, 2012.

\begin{tabular}{|c|c|c|c|c|c|c|c|}
\hline \multirow{3}{*}{\multicolumn{2}{|c|}{ Variáveis de investigação: ações de matriciamento }} & \multicolumn{6}{|c|}{$\begin{array}{c}\text { Construção conjunta dos Projetos Terapêuticos } \\
\text { Singulares }\end{array}$} \\
\hline & & \multirow{3}{*}{$\begin{array}{c}\text { Sim } \\
\% \text { Col } \\
\text { N1 }= \\
9.057\end{array}$} & \multirow{3}{*}{$\begin{array}{c}\text { Não } \\
\% \text { Col } \\
\text { N2= } \\
6.063\end{array}$} & \multirow{3}{*}{$\begin{array}{c}\text { Total } \\
\% \text { Col } \\
\text { N1+N2= } \\
15.120\end{array}$} & \multirow{3}{*}{$\mathrm{OR}^{*}$} & \multicolumn{2}{|c|}{ IC $95 \%$} \\
\hline & & & & & & & \\
\hline \multicolumn{2}{|r|}{ Estratégias de cuidado } & & & & & Inferior & Superior \\
\hline \multirow{14}{*}{ 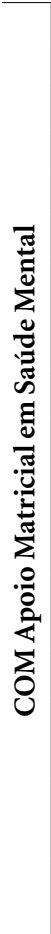 } & Qualificação da Clínica & 65,4 & 45,0 & 57,5 & 2,30 & 2,11 & 2,51 \\
\hline & Consulta específica com tempo maior & 52,1 & 34,8 & 45,4 & 2,04 & 1,87 & 2,23 \\
\hline & Registro da história de vida & 57,7 & 34,9 & 48,9 & 2,54 & 2,33 & 2,78 \\
\hline & Desenvolvimento de novas tecnologias & 81,9 & 59,3 & 73,1 & $3,10^{\star *}$ & 2,82 & 3,41 \\
\hline & Oferta de algum tipo de atendimento em grupo & 44,9 & 18,3 & 34,6 & $3,64^{\star *}$ & 3,29 & 4,03 \\
\hline & Atendimento com profissionais de saúde mental & 75,7 & 53,5 & 67,1 & 2,71 & 2,48 & 2,97 \\
\hline & $\begin{array}{l}\text { A equipe realiza ações para pessoas que fazem } \\
\text { uso crônico de benzodiazepínicos e necessitam } \\
\text { desmame (diminuição da dose)? }\end{array}$ & 32,9 & 16,2 & 26,4 & 2,64 & 2,36 & 2,94 \\
\hline & Gestão do Caso & 66,5 & 38,7 & 55,7 & $3,15^{\star *}$ & 2,89 & 3,44 \\
\hline & $\begin{array}{l}\text { A equipe possui protocolos com definição } \\
\text { de diretrizes terapêuticas para acolhimento à } \\
\text { demanda espontânea/urgência para problemas } \\
\text { relacionados à saúde mental? }\end{array}$ & 85,9 & 69,4 & 81,7 & 2,68 & 2,27 & 3,16 \\
\hline & $\begin{array}{l}\text { A eSF teve preparação para o atendimento dos } \\
\text { usuários com transtorno mental? }\end{array}$ & 46,9 & 25,7 & 38,7 & 2,55 & 2,32 & 2,80 \\
\hline & Qualificação do registro & 71,2 & 53,6 & 64,3 & 2,14 & 1,95 & 2,33 \\
\hline & $\begin{array}{l}\text { A eSF possui registro dos casos mais graves de } \\
\text { usuários com transtorno mental? }\end{array}$ & 53,6 & 33,9 & 46,0 & 2,25 & 2,06 & 2,46 \\
\hline & $\begin{array}{l}\text { Há registro de usuários c/ necessidades por uso } \\
\text { de crack/álcool/outras drogas? }\end{array}$ & 33,5 & 17,9 & 27,4 & 2,31 & 2,08 & 2,56 \\
\hline & $\begin{array}{l}\text { A equipe possui registro dos usuários em uso } \\
\text { crônico de benzodiazepínicos? }\end{array}$ & 52,4 & 38,9 & 47,2 & 1,73 & 1,58 & 1,88 \\
\hline
\end{tabular}

Fonte: Elaboração própria com base no Programa Nacional de Melhoria do Acesso e da Qualidade da Atenção Básica (PMAQ-AB), em seu primeiro ciclo de aplicação (2012).

especializados. Por fim, na categoria DNT, as intervenções para diminuição de doses de benzodiazepínicos são as menos afetadas, entretanto, ainda assim realizadas pelo menos duas vezes mais na presença do AMSM. Segundo dados do Sistema Nacional de Gerenciamento de Produtos Controlados (SNGPC), de 2007 a 2010, tal classe de medicamento controlado foi a mais utilizada pela população brasileira ${ }^{25}$ e como seu uso inadequado e prolongado pode causar dependência, transformou-se em um problema de saúde pública no país.

Na Gestão do Caso, em nível "desagregado", a diferença entre o efeito nas estratégias de cuidado é sutil. Destaca-se a preparação das equipes de $\mathrm{AB}$ para o atendimento a usuários com transtorno mental como estratégia mais implementada, sofrendo maior influência positiva das ações 2 (discussão de casos) e 3 (ações clínicas compartilhadas). A existência de diretrizes para o acolhimento inicial às demandas de saúde mental se associou positivamente, embora com menor intensidade, à ação 4 (construção conjunta do PTS).

Os efeitos nestas estratégias de cuidado são particularmente importantes, pois interferem diretamente na capacidade resolutiva da $A B$ nos casos de SM e são determinantes na adesão ou não do usuário ao tratamento. Os dados evidenciam que o AM está conseguindo de fato fornecer subsídios aos profissionais da $\mathrm{AB}$ para que possam atuar com mais qualidade nos casos de SM, auxiliando na superação da falta de capacitação dos profissionais da $\mathrm{AB}$. A existência de diretrizes terapêuticas voltadas para o acolhimento é essencial. Trata-se de uma ação técnico-assistencial ${ }^{26}$ 
com a proposta de qualificação da escuta e início da construção de vínculo dos usuários com os profissionais e serviços de saúde, o que faz total diferença para a adesão às propostas terapêuticas.

A Qualificação da Clínica, por sua vez, sofre maior influência das ações 2 (discussão de casos) e 3 (ações clínicas compartilhadas), respectivamente, seguida da ação 4 (construção conjunta do PTS), porém com diferença pequena na força da interferência entre estas ações. No nível "desagregado" o registro mais detalhado sobre a história de vida dos usuários sofreu maior influência do que o maior tempo de consulta, na ocorrência das três ações.

A Qualificação do Registro, em comparação às outras três categorias de estratégias de cuidado, é a que demonstra menor efeito no desempenho, porém com associação positiva com todas as ações. Sob influência das três ações destacadas de AMSM os registros são feitos duas vezes mais e tal padrão é visto também na análise desagregada desta estratégia de cuidado. A exceção está no registro das pessoas que fazem uso crônico de benzodiazepínicos, sendo realizado apenas uma vez mais.

O registro é algo altamente importante pois permite saber a situação de saúde dos indivíduos, famílias e território e fornece subsídios para que as ações sejam direcionadas para cada público alvo de maneira mais efetiva ${ }^{27}$. Saber a quantidade de casos com transtorno mental grave, de pessoas que fazem uso prejudicial de algum tipo de droga e de benzodiazepínicos, por exemplo, auxilia na tomada de decisões sobre quais estratégias de cuidado são necessárias.

Todas as estratégias de cuidados são potencializadas sob a influência das ações de AMSM. De modo geral, tanto em nível "agregado" como “desagregado", são implementadas de duas a três vezes mais. As três ações investigadas de maneira mais minuciosa apontaram seus efeitos relevantes, na qualificação do cuidado. Cabe, contudo, salientar que tanto a discussão de casos (ação 2), como a construção conjunta do PTS (ação 4) são atividades que fazem parte das ações clínicas compartilhadas. Este resultado demonstra que o AMSM se faz mais eficaz quando envolve o trabalho conjunto entre os profissionais da $\mathrm{AB}$ e da saúde mental, pensando e atuando nos casos, e superam as atividades de educação permanente quando realizadas isoladamente.

Os resultados deste estudo confirmam e geram evidências de que o trabalho integrado e o modelo de cuidados colaborativos, com o desenvolvimento de ações compartilhadas de maneira interdisciplinar é o que realmente se traduz em efeitos na qualificação do cuidado em SM na AB. Foram utilizados para tal, de maneira inédita até o momento, dados obtidos através da aplicação do principal instrumento avaliativo da $\mathrm{AB}$ do Brasil. O presente estudo traz como inovação a investigação quantitativa acerca dos efeitos do apoio matricial no cuidado ao usuário e a utilização dos dados do PMAQ-AB para tal.

É importante ponderar que o PMAQ-AB é um instrumento orientado para balizar as ações de gestão. Com isso, a investigação voltada para o cuidado utilizando seus dados foi realizada a partir da utilização de variáveis de diferentes partes do mesmo. Além disso, destaca-se que os resultados aqui obtidos, acerca do impacto positivo no cuidado em SM na $\mathrm{AB}$ devido ao AM, já eram esperados, considerando a literatura científica. Este estudo, utilizando abordagem quantitativa, comprova as proposições teóricas e ajuda a reforçar o modelo de cuidados colaborativos.

Contudo, verificou-se que as ações de matriciamento ainda estão sendo implementadas de maneira insuficientes nas diferentes unidades do SUS. Os resultados apresentados demonstram que o cuidado básico em saúde mental se faz presente somente de $55 \%$ a $73 \%$ das equipes. A amplitude da ocorrência de transtornos mentais na população e o entendimento que todas as ações de cuidado em saúde envolvem o cuidado em saúde mental aponta que este não pode ser configurado como um campo em separado. Soma-se a esta análise o fato de que os resultados são obtidos pelas respostas dos profissionais da AB. Frente a um cenário de precarização do SUS e diante de um sistema avaliativo que está associado a obtenção de recursos financeiros para o serviço, há que se considerar a possibilidade de um viés que supervalorize as respostas fornecidas.

Entende-se ainda como limitações deste estudo a impossibilidade de comparação entre os ciclos do PMAQ-AB, uma vez que as questões relacionadas ao apoio matricial sofreram alterações substanciais que prejudicaram a qualidade da investigação. Neste aspecto, ressalta-se a investigação do uso de psicotrópicos. Em 2012, há uma variável sobre o registro de usuários que fazem uso crônico de benzodiazepínicos e outra acerca do cuidado na redução de doses. Contudo, em 2014, há um grande equívoco metodológico, pois é investigado na mesma variável classes muito diferentes de medicamentos, quais sejam: benzodiazepínicos, antipsicóticos, anticonvulsivantes, antidepressivos, estabilizadores de humor e ansiolíticos. $\mathrm{Na}$ análise desta questão pressu- 
põem-se que todos os psicotrópicos têm a mesma indicação clínica, o mesmo padrão ouro de uso e induzem dependência da mesma forma, o que se configura em um grande equívoco teórico sobre o tema.

Este estudo apresenta limitações em seu escopo, pois não abordamos as avaliações de usuários ou de profissionais dos CAPS e NASF. A falta de informações acerca da percepção dos usuários sobre as ações de matriciamento e o cuidado em $S M$ na $A B$, baseia-se no fato de as perguntas do PMAQ-AB direcionadas aos usuários se referirem apenas à questões de acesso, não havendo no instrumento questões específicas para qualificação da clínica/cuidado em SM sob a perspectiva do usuário. E a falta de avaliação do AM desenvolvidas pelos CAPS e NASF a partir das respostas dos profissionais destas unidades só foi realizado em 2014, não fazendo parte do escopo do estudo.

É de suma importância que mais estudos sejam realizados abordando a temática do apoio matricial através de metodologia quantitativa, pois a ausência destes dados enfraquece a análise do modelo de cuidado e a avaliação de sua implementação nas políticas públicas. Tal constatação se evidenciada nas mudanças da proposta de trabalho atual do Nasf, com redução significativa da sua força de trabalho, justificada pela ausência de evidências de sua efetividade. Há que se acrescentar que o PMAQ-AB é o único instrumento de abrangência nacional que avalia de maneira tão extensa a política de atenção básica e seu objetivo de avaliação fica frágil se os dados não são explorados em sua potência máxima.

\section{Colaboradores}

Todos os autores contribuíram na análise e interpretação dos dados, na elaboração do artigo e na aprovação da versão final a ser publicada.

\section{Referências}

1. Schramm JM de A, Oliveira AF de, Leite I da C, Valente JG, Gadelha ÂMJ, Portela MC, Campos MR. Transição epidemiológica e o estudo de carga de doença no Brasil. Cien Saude Colet 2004; 9(4):897-908.

2. Organização Pan-americana da Saúde (OPAS), Organização Mundial da Saúde (OMS). Relatório sobre a saúde no mundo 2001: saúde mental: nova concepção, nova esperança. Genebra: OPAS/OMS; 2001.

3. World Health Organization, Department of Mental Health and Substance Abuse. Mental Health Gap Action Programme: scaling up care for mental, neurological, and substance use disorders. Geneva: WHO Press; 2008.

4. Poletto AR, Gontijo LA. Integração da saúde mental nos cuidados de saúde primários: Uma perspectiva global. 2008 [acessado 2017 out 5]; Disponível em: http://www.abepro.org.br/biblioteca/enegep2013_ TN_STO_180_026_23287.pdf

5. Giovanella L, organizador. Políticas e sistema de saúde no Brasil. $2^{\text {a }}$ ed. rev. e ampliada. Rio de Janeiro: Editora Fiocruz; Centro Brasileiro de Estudos da Saúde; 2012.

6. Starfield B. Atenção primária: equilíbrio entre necessidades de saúde, serviços e tecnologia. Brasília: UNESCO; 2002.

7. Gonçalves DA, Mari JJ, Bower P, Gask L, Dowrick C, Tófoli LF, Campos M, Portugal FB, Ballester D, Fortes S. Estudo multicêntrico brasileiro sobre transtornos mentais comuns na atenção primária: prevalência e fatores sociodemográficos relacionados. Cad Saude Publica 2014; 30(3):623-632. 
8. Lima FG, Siciliani CC, Drehmer LBR. O perfil atual da saúde mental na atenção primária brasileira. Com Cien Saude 2012; 24(2):143-148.

9. Figueiredo MD, Campos RO. Saúde Mental na atenção básica à saúde de Campinas, SP: uma rede ou um emaranhado? Cien Saude Colet 2009; 14(1):129-138.

10. Chazan LF, Impagliazzo SP, Silva AM, Alves KVG, Albuquerque AB, Ciuffo RS. Trabalho colaborativo em saúde mental. Rev Hosp Univ Pedro Ernesto [Internet]. 2016 [acessado 2018 out 31]; 15(3): [cerca de 7 p.]. Disponível em: http://www.e-publicacoes.uerj.br/index.php/revistahupe/article/view/29453

11. Ivbijaro GO, Enum Y, Khan AA, Lam SS-K, Gabzdyl A. Collaborative Care: Models for Treatment of Patients with Complex Medical-Psychiatric Conditions. Curr Psychiatry Rep [Internet]. 2014 [cited 2018 Sep 23]; 16(11): [about 24p.]. Available from: http://link. springer.com/10.1007/s11920-014-0506-4

12. Kates N. Mental Health and Primary Care: Contributing to Mental Health System Transformation in Canada. Can J Commun Ment Health [Internet] Oct 2018 [cited 2018 Dec 15]; 36(4): [about 35 p.]. Available from: https://www.cjcmh.com/doi/pdf/10.7870/ cjcmh-2017-033

13. Campos GWS, others. Equipes de referência e apoio especializado matricial: um ensaio sobre a reorganização do trabalho em saúde. Cien Saude Colet [Internet]. 1999 [acessado 2017 out 5]; Disponível em: http://www.scielo.br/scielo.php?pid=s1413 $-81231999000200013 \&$ script $=$ sci_abstract\&tlng $=$ pt

14. Campos GW S, Domitti AC. Apoio matricial e equipe de referência: uma metodologia para gestão do trabalho interdisciplinar em saúde. Cad Saude Publica 2007; 23(2): 399-407.

15. Chiaverini DH, Gonçalves DA, Ballester D, Tófoli LF, Chazan LF, Almeida N, Fortes S. Guia prático de matriciamento em saúde mental [Internet]. Ministério da Saúde, Centro de Estudo e Pesquisa Coletiva [Internet]. 2011 [acessado 2017 out 23]. Disponível em: http://bvsms.saude.gov.br/bvs/publicacoes/guia_pratico_matriciamento_saudemental.pdf

16. Brasil. Portaria $\mathrm{n}^{\circ} 154$, de 24 de janeiro de 2008. Cria os Núcleos de Apoio à Saúde da Família - NASF. Diário Oficial da União, 2008; 24 jan.

17. Brasil. Ministério da Saúde (MS). Secretaria de Atenção à Saúde, Departamento de Ações Programáticas Estratégicas. Núcleo de Apoio à Saúde da Família: ferramentas para a gestão e para o trabalho cotidiano. Cad Aten Basica 2014;1:1-118.

18. Jorge MA, Carvalho MCA, Silva PRF, organizadores. Políticas e cuidado em saúde mental: contribuições para a prática profissional. Rio de Janeiro: Editora Fiocruz; 2014. $295 \mathrm{p}$.

19. Pinto HA, Sousa Ana, Ferla AA. O Programa Nacional de Melhoria do Acesso e da Qualidade da Atenção Básica: faces de uma política inovadora. Saude Debate [Internet]. 2014 [acessado 2018 out 30]; v.38 (especial): 14 p. Disponível em: http://www.gnresearch. org/doi/10.5935/0103-1104.2014S027
20. Filho JM, Silveira LMC. Consulta Conjunta: uma Estratégia de Capacitação para a Atenção Integral à Saúde. Rev Brasil Educ Medica [Internet] 2005 mai -ago [acessado 2017 dez 20]; v.29: 5 p. Disponível em: http://www.educacaomedica.org.br/UserFiles/ File/2005/volume29_2/consulta_conjunta.pdf

21. Fortes S, Furlanetto LM, Chazan LF. Modelo para a implantação de interconsulta e consulta conjunta com a equipe do Programa de Saúde da Família (PSF). Associação Brasileira de Psicanálise [Internet] 2005 [acessado 2018 nov 6]; Disponível em: http://www. congressoabp.org.br/boletim/exibBoletim/?bol_ id $=4 \&$ boltex_id $=20$

22. Brasil. Ministério da Saúde (MS). Núcleo Técnico da Política Nacional de Humanização. Clínica ampliada, equipe de referência e projeto terapêutico singular. $2^{\mathrm{a}}$ ed. Brasília: MS; 2008.

23. Carvalho SR.; Cunha GT. A gestão da atenção na saúde: elementos para se pensar a mudança da organização na saúde. In: Campos GWS, organizador. Tratado de saúde coletiva. São Paulo: Hucitec; 2006. p. 837-868.

24. Minozzo F, Kammzetser CS, Debastiani C, Fait CS, Paulon SM. Grupos de saúde mental na atenção primária à saúde. Fractal Rev Psicol 2012; 24(2):323-340.

25. Sistema Nacional de Gerenciamento de Produtos Controlados. Boletim de Farmacoepidemiologia. Agência Nacional de Vigilância Sanitária [Internet]. 2011 [acessado $2018 \mathrm{dez}$ 10]; 2: 9 p. Disponível em: http:// www.anvisa.gov.br/sngpc/boletins/2011/boletim_sngpc_2edatualizada.pdf

26. Núcleo Técnico da Política Nacional de Humanização. Acolhimento nas práticas de produção de saúde. Brasília: Editora MS; 2009.

27. Fraga L, Carneiro CCG. [Resenha]. Saúde coletiva: teoria e prática. Paim JS, Almeida-Filho N, organizadores. $1^{\text {a }}$ ed. Rio de Janeiro: Medbook; 2014.720 p. Trab Educ Saude 2016; 14(1):313-315.

Artigo apresentado em 29/04/2019

Aprovado em 23/08/2019

Versão final apresentada em 25/08/2019

Editores-chefes: Romeu Gomes, Antônio Augusto Moura da Silva 